\title{
Neumocistosis en pacientes venezolanos: diagnóstico y epidemiología (2001-2006)
}

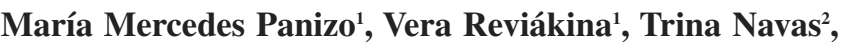
Karina Casanova ${ }^{2}$, Alejandro Sáez ${ }^{2}$, Rafael Napoleón Guevara ${ }^{3,4}$, Ana María Cáceres ${ }^{4}$, Raúl Vera ${ }^{5}$, Carlos Sucre $^{5}$ y Esther Arbona ${ }^{5}$

${ }^{\prime}$ Departamento de Micología, Instituto Nacional de Higiene Rafael Rangel; ${ }^{2}$ Servicio de Medicina Interna, Hospital General del Oeste Dr. José Gregorio Hernández; ${ }^{3}$ Servicio de Enfermedades Infecciosas del Adulto, Hospital Universitario de Caracas; ${ }^{4}$ Unidad de Infectología, Instituto Médico La Floresta; ${ }^{5}$ Unidad de Oncología Médica, Instituto Médico La Floresta, Caracas, República Bolivariana de Venezuela

Resumen El objetivo de este trabajo fue conocer la epidemiología de la neumocistosis en pacientes venezolanos mediante un estudio retrospectivo en un período de seis años. Se procesaron 129 muestras clínicas de pacientes con sida, cáncer, e infección respiratoria de vías bajas sin sida ni cáncer, por la técnica de inmunofluorescencia directa. Se diagnosticaron 30 casos de neumocistosis $(23,3 \%)$, y la frecuencia de diagnóstico varió según el grupo de pacientes: $36,6 \%$ en pacientes con sida, $38 \%$ en pacientes con cáncer y $10,4 \%$ en pacientes con infección respiratoria de vías bajas sin sida ni cáncer. Este estudio demostró que existen diferencias en la presentación de la neumocistosis relacionada con la enfermedad de base de los pacientes, y que esta enfermedad es un problema de salud importante en individuos inmunosuprimidos en Venezuela. La neumocistosis debe ser sospechada en pacientes no inmunosuprimidos con signos y síntomas de infección respiratoria de vías bajas, y se debe ampliar el estudio de esta enfermedad en pacientes con cáncer y enfermedad pulmonar obstructiva crónica. La inmunofluorescencia directa es una técnica sumamente útil para el diagnóstico de la neumocistosis y sus resultados dependen de una muestra en óptimas condiciones y de la experiencia del personal de laboratorio a cargo del diagnóstico.

Palabras clave Neumocistosis, Cáncer, Enfermedad pulmonar obstructiva crónica, Sida, Inmunofluorescencia directa

\section{Pneumocystosis in Venezuelan patients: epidemiology and diagnosis (2001-2006)}

Summary The objective of this work was to investigate the epidemiology of pneumocystosis in Venezuelan patients utilizing a retrospective study during a six year period. One hundred and twenty nine clinical samples collected from patients with AIDS, cancer and non-AIDS-non-cancer low respiratory tract infection patients were processed by direct immunofluorescence technique. Pneumocystosis was diagnosed in 30 patients with a general frequency of $23.3 \%$, which varied according to the patient's group: $36.6 \%$ in AIDS patients, $38 \%$ in cancer patients, and $10.4 \%$ in non-AIDS-non-cancer low respiratory tract infection patients. This study demonstrated the existence of differences in pneumocystosis frequency related to the patient's underlying disease, and that the illness is an important health problem in immunocompromised patients in Venezuela. Pneumocystosis must be suspected in non-immunocompromised patients with signs and symptoms of low respiratory tract infection, and the study of this illness must include COPD and cancer patients. Direct immunofluorescence is a useful technique for pneumocystosis diagnosis, however, it requires an optimal sample and skilled personnel in the laboratory.

Key words Pneumocystosis, Cancer, Chronic obstructive pulmonary disease, AIDS, Direct immunofluorescence 
Pneumocystis jirovecii, conocido anteriormente como Pneumocystis carinii sp. f. hominis, es el agente causal de la neumocistosis, una infección oportunista que causa neumonía grave en pacientes inmunodeficientes. La neumocistosis es considerada una enfermedad definitoria de la presencia del síndrome de inmunodeficiencia adquirida (sida) en pacientes infectados con el virus de la inmunodeficiencia humana (VIH), pero los casos de esta enfermedad en individuos con otros factores predisponentes y condiciones de inmunosupresión diferentes al VIH, como el cáncer, trasplante de órganos y enfermedades hematológicas, entre otras, están aumentando progresivamente $[2,6,11,19,24,32]$.

Diagnosticar la neumocistosis es un reto desde el punto de vista clínico y microbiológico. Esto se debe principalmente a la presencia de signos y síntomas inespecíficos, el uso de profilaxis en los pacientes con VIH y la presencia de tuberculosis e infección por citomegalovirus en los pacientes inmunodeficientes. Por otra parte, la ausencia de un sistema in vitro para cultivar el microorganismo, el hecho de que es especie-específico, que las muestras necesarias para su diagnóstico no son fáciles de obtener debido a las condiciones de los pacientes y que se necesita personal cualificado en el procesamiento e interpretación de los resultados de las pruebas diagnósticas, ha hecho difícil el estudio de $P$. jirovecii desde el punto de vista microbiológico $[9,17,32]$.

En Venezuela, los estudios sobre la neumocistosis son escasos, por lo que no se conoce bien la epidemiología de la enfermedad $[3,7,8,25]$. Esto se debe principalmente a que no se incluye la neumocistosis como diagnóstico diferencial, sobre todo en pacientes inmunodeficientes sin VIH o en pacientes con largos tratamientos con antibióticos, drogas citotóxicas y corticosteroides sin evolución adecuada postratamiento, así como a la ausencia de diagnóstico micológico en los centros hospitalarios.

Por estas razones, el objetivo de este trabajo fue conocer la epidemiología de la neumocistosis en pacientes venezolanos mediante un estudio retrospectivo de esta patología durante seis años, en el Departamento de Micología del Instituto Nacional de Higiene Rafael Rangel.

\section{Materiales y métodos}

Se realizó un estudio de corte transversal y retrospectivo de revisión de historias micológicas en el Departamento de Micología del Instituto Nacional de Higiene Rafael Rangel (INHRR). Los pacientes en estudio, dentro del período comprendido entre enero de 2001 y diciembre de 2006, tenían un diagnóstico presuntivo de neumocistosis. Todos los pacientes presentaron sintomatología pulmonar aguda y cumplieron con los criterios de inclusión, dividiéndose en tres grupos: i) pacientes con sida; ii) pacientes con cáncer (tumores sólidos, linfomas y leucemias); iii) pacientes con infección respiratoria de vías bajas sin sida ni cáncer (IRB): presencia de enfermedades autoinmunes, insuficiencia renal, hepatopatías graves (agudas o crónicas), enfermedades pulmonares crónicas, deficiencias nutricionales protéico-calóricas, infección respiratoria pulmonar aguda de cualquier tipo, y pacientes en postoperatorio de cirugías mayores, complicadas o no, con infecciones nosocomiales.

Los datos demográficos, las enfermedades de base de los pacientes y los tratamientos se recopilaron en las historias micológicas.
Se procesaron diversas muestras clínicas que consistieron en 89 esputos espontáneos, 15 esputos inducidos, 24 lavados bronquiales y una biopsia de pulmón.

Se realizó la técnica de inmunofluorescencia directa con la prueba Merifluor® Pneumocystis (Meridian Bioscience, Inc.), diseñada para la detección in vitro de quistes (ascas) y trofozoítos (ascosporas) de $P$. jirovecii en muestras provenientes del tracto respiratorio. El reactivo de detección contiene anticuerpos monoclonales marcados con isotiocianato de fluoresceína, dirigidos contra la pared celular y los antígenos de la matriz de $P$. jirovecii $[12,15]$. Se siguieron las instrucciones del fabricante para el procesamiento de las muestras de esputo inducido, lavado bronquial y biopsias, con algunas modificaciones en el procesamiento de las muestras de esputo espontáneo, estandarizadas en el Departamento de Micología del INHRR.

La técnica de procesamiento para las muestras de lavado bronquial consiste en concentrar la muestra por centrifugación, descartar todo el sobrenadante menos $0,5 \mathrm{ml}$ y resuspender el sedimento. Posteriormente, se toman $25 \mu \mathrm{l}$ de la muestra y se colocan en el pozo de una lámina portaobjeto para pruebas de inmunofluorescencia. Se deja secar al aire, se fija con acetona durante $10 \mathrm{~min}$ y se deja secar nuevamente. Se coloca la lámina en una cámara húmeda, se agrega el reactivo de detección y se incuba durante $30 \mathrm{~min}$ en estufa a $35^{\circ} \mathrm{C}$. Al finalizar el tiempo de exposición, las láminas son lavadas con agua para eliminar el exceso de reactivo, se remueve el exceso de agua $y$, sin dejar que la lámina se seque completamente, se añade el líquido de montaje, se coloca un cubreobjetos y se examina inmediatamente con el microscopio de fluorescencia a una longitud de onda de 490-500 nm y objetivo de 40x. Para las muestras de esputo inducido, se realiza un frotis de la muestra directamente en el pozo de la lámina de inmunofluorescencia. En las muestras de biopsia, se corta un trozo de la misma y se realiza un frotis por aposición directamente en el pozo de la lámina portaobjeto. Posteriormente, se sigue el procedimiento descrito anteriormente para ambas muestras citadas $[12,15]$.

Para las muestras de esputo espontáneo, la modificación consistió en realizar de tres a cinco lavados sucesivos de la muestra con solución salina tamponada con fosfatos (PBS) a pH 7,2 en dilución 1:10 (1 ml de esputo por $9 \mathrm{ml}$ de PBS), seguido de homogeneización con vórtex y centrifugación a $3.500 \mathrm{rpm}$ durante $10 \mathrm{~min}$, con la finalidad de reducir su viscosidad y obtener una mayor concentración del microorganismo. Se sigue el procedimiento descrito anteriormente, pero aumentando el tiempo de exposición del esputo diluido al reactivo de detección en $75 \min [3,25]$.

Como control positivo se utilizó una muestra de lavado broncoalveolar de un paciente con sida con diagnóstico de neumocistosis por coloración de Gomori-Grocott y por inmunofluorescencia directa.

Para la interpretación de los resultados, se siguieron las instrucciones del fabricante: cualquier muestra que tuviera dos quistes típicos o más con fluorescencia verde manzana y morfología característica se consideró positiva para la presencia de $P$. jirovecii. Por lo general, los quistes (ascas) suelen encontrarse junto con trofozoítos (ascosporas) en racimos que pueden variar de tamaño y presentar, o no, estructuras que asemejan un panal de abejas. Las muestras que no presentaron las características descritas anteriormente se consideraron negativas $[12,15]$.

Para el análisis de los resultados se calculó la frecuencia porcentual de neumocistosis en general y en cada grupo de pacientes. Los datos continuos se describieron usando medidas de tendencia central. 


\section{Resultados}

Se estudiaron 129 pacientes con sintomatología pulmonar aguda y sospecha clínica de neumocistosis en un período de seis años. De estos pacientes, 41 tenían sida (31,8\%), 21 algún tipo de cáncer $(16,3 \%)$ y 67 IRB $(51,9 \%)$. Fue notable el predominio del género masculino en los pacientes evaluados: 93 del género masculino $(74,4 \%)$ y 36 del género femenino $(25,6 \%)$, con una relación hombre-mujer de 3:1. La edad promedio fue de 49,5 $\pm 17,9$ años, siendo la edad promedio de los hombres $50 \pm 19$ años y la de las mujeres 48,6 \pm 15,3 años.

Se detectaron 30 casos de neumocistosis mediante inmunofluorescencia directa, con una frecuencia general para la enfermedad de 23,3\% (30 de 129), que varió según el grupo de pacientes: 36,6\% (15 de 41) en pacientes con sida, $38 \%$ ( 8 de 21 ) en pacientes con cáncer y $10,4 \%$ ( 7 de 67 ) en pacientes con IRB.

En el grupo de pacientes con sida la edad promedio fue de $38,70 \pm 11,39$. No fue posible la obtención de datos tales como tratamiento antirretroviral, contaje de subpoblaciones linfocitarias $\mathrm{CD}^{+} / \mathrm{CD}^{+}$y tratamiento empírico con antimicrobianos. Se presentaron dos casos de neumocistosis con candidosis oral y uno asociado a la infección por citomegalovirus. Sólo se pudo documentar el tratamiento antirretroviral en un paciente, y tratamiento antibacteriano de amplio espectro en tres casos.

En los pacientes con cáncer, la edad promedio fue $52,16 \pm 14,86$. Los factores predisponentes más importantes fueron la presencia de leucemia linfocítica, seguida de linfoma de Hodgkin y tumores sólidos, además del tratamiento con antibióticos de amplio espectro y la quimioterapia (Tabla 1 ).

En el grupo de pacientes con IRB la edad promedio fue $52,38 \pm 18,92$. Se detectaron siete casos de neumocistosis, de los cuales dos presentaron neumonía y cinco tenían enfermedad pulmonar obstructiva crónica (EPOC) acompañada con cor pulmonale (un caso), cardiopatía isquémica e hipertensión arterial (un caso), artritis reumatoide (un caso, diagnosticado por biopsia), hepatopatía alcohólica (un caso) y neumonía (un caso). Los dos primeros casos con EPOC tenían tratamiento con antibacterianos de amplio espectro, el paciente con artritis reumatoide estaba en tratamiento con esteroides y metotrexato, y los pacientes con neumonía y hepatopatía alcohólica tenían tratamiento antituberculoso.

La distribución del tipo de muestras recibidas para el diagnóstico de neumocistosis por grupo de pacientes se presenta en la tabla 2. La muestra recibida con mayor frecuencia fue el esputo espontáneo en los grupos de pacientes con sida e IRB. En cambio, en pacientes con cáncer, la muestra recibida con mayor frecuencia fue el lavado broncoalveolar. De 89 esputos espontáneos procesados, en 12 se detectó la presencia de $P$. jirovecii, particularmente en

Tabla 1. Características de los pacientes con cáncer $(n=21)$.

\begin{tabular}{lc}
\hline Características & $N^{\circ} /$ total \\
\hline Pacientes estudiados & $21 / 129$ \\
Neumonía por $P$. jirovecii & 8 / 21 \\
Leucemia linfocítica & $2 / 2$ \\
Linfoma de Hodgkin & $3 / 4$ \\
Tumores sólidos & $3 / 15$ \\
Neumonía por $P$. jirovecii y tratamiento & $8 / 21$ \\
Tratamiento con esteroides & $1 / 8$ \\
Quimioterapia & $4 / 8$ \\
Tratamiento con antibacterianos de amplio espectro & $3 / 8$
\end{tabular}

pacientes con sida. De 15 esputos inducidos, en 11 se detectó la presencia del microorganismo, siendo una muestra útil para el diagnóstico de la enfermedad en los grupos de pacientes con sida e IRB. De 24 muestras de lavado broncoalveolar recibidas, en seis se detectó la presencia de $P$. jirovecii, en su mayoría provenientes de pacientes con cáncer.

\section{Discusión}

Existen diferencias en la presentación de la neumocistosis que dependen de la enfermedad de base de los pacientes. En el grupo de pacientes con sida la neumocistosis se presenta con más frecuencia, pero en los pacientes con cáncer la frecuencia también es elevada y no se deben despreciar los resultados obtenidos en el grupo de pacientes con IRB sin sida ni cáncer.

La frecuencia de la neumocistosis varía dependiendo del grupo de pacientes, el período de tiempo, la técnica empleada para el diagnóstico y el país donde se realiza el estudio [2,3,6,7,11,14,19,24,27]. En la mayoría de las referencias bibliográficas revisadas, los pacientes estudiados se dividen en dos grandes grupos: con infección por el VIH y sin infección por el VIH, incluyendo en este último los pacientes con cáncer y con diferentes condiciones clínicas $[6,14]$; en otras investigaciones se separaron los grupos con infección por el VIH/sida $[3,11,19]$ y con cáncer [2,24,27], observándose que la frecuencia de neumocistosis varió dependiendo del tipo de cáncer. Hay pocos estudios que separan a los pacientes con inmunosupresión por sida y cáncer de los pacientes con otras enfermedades de base $[7,8]$, por lo que se hace difícil establecer comparaciones. Pensamos que los pacientes con enfermedades de base diferentes al sida y el cáncer deben estudiarse por separado.

La presencia de coinfecciones asociadas a la neumocistosis en pacientes con sida debe ser un dato importante a tener en cuenta al evaluar esta enfermedad. La infección por citomegalovirus puede ser causa de deficiencias respiratorias en estos pacientes, complicando su evolución clínica. La candidosis oral, por su parte, es un factor de riesgo para el desarrollo de neumocistosis y se considera una enfermedad definitoria de la presencia de sida. Por lo tanto, se sugiere que las muestras del tracto respiratorio y otras muestras apropiadas sean enviadas al laboratorio para la detección de otros patógenos bacterianos, fúngicos y virales. El reconocimiento de la presencia de lesiones orales, así como de otras infecciones, podría indicar la necesidad de iniciar vigilancia intensiva de la aparición de las mismas (tanto clínica como microbiológica), así como el inicio de profilaxis $[14,23,34]$.

Según los datos de nuestro estudio, en valores absolutos, en el grupo con cáncer la neumocistosis se presentó con mayor frecuencia en los pacientes con neoplasias hematológicas, seguida de linfoma de Hodgkin y tumores sólidos. Aunque el grupo de pacientes con cáncer es pequeño, estos datos son diferentes a los obtenidos por otros autores, en los que el linfoma de Hodgkin seguido de las leucemias, son los principales factores de riesgo para la enfermedad [2,24,27], mientras que los tumores sólidos se encuentran en el tercer o cuarto lugar de la lista, resultado similar al obtenido en este trabajo. La frecuencia de neumocistosis en pacientes con tumores sólidos generalmente es baja, pero puede aumentar dependiendo de la intensidad de la quimioterapia. En nuestro trabajo, la mitad de los pacientes con cáncer y neumocistosis recibían este tratamiento. Resultados similares fueron obtenidos en otras investigaciones [6,10,28,31]. 
Tabla 2. Distribución del tipo de muestras empleadas para el diagnóstico de neumocistosis por grupo de pacientes.

\begin{tabular}{|c|c|c|c|c|c|c|c|c|}
\hline \multirow{3}{*}{ Tipo de muestras } & \multicolumn{8}{|c|}{ Grupo de pacientes } \\
\hline & \multicolumn{2}{|c|}{ Sida } & \multicolumn{2}{|c|}{ Cáncer } & \multicolumn{2}{|c|}{ IRB } & \multicolumn{2}{|c|}{ Total } \\
\hline & MP & $\mathrm{Pj}+$ & MP & $\mathrm{Pj}+$ & MP & $\mathrm{Pj}+$ & MP & $\mathrm{Pj}+$ \\
\hline Esputo espontáneo & 33 & 10 & 4 & 2 & 52 & - & 89 & 12 \\
\hline Esputo inducido & 6 & 5 & 3 & 1 & 6 & 5 & 15 & 11 \\
\hline Lavado broncoalveolar & 2 & - & 14 & 5 & 8 & 1 & 24 & 6 \\
\hline Biopsia de pulmón & - & - & - & - & 1 & 1 & 1 & 1 \\
\hline Total & 41 & 15 & 21 & 8 & 67 & 7 & 129 & 30 \\
\hline
\end{tabular}

MP: muestras procesadas; $\mathrm{Pj}+$ : muestras positivas para diagnóstico de $P$. jirovecii por inmunofluorescencia directa.

En los pacientes con IRB el hallazgo más importante fue la presencia de neumocistosis con EPOC, y todos ellos, además, presentaron enfermedades asociadas muy importantes. Las investigaciones que han tratado de relacionar la presencia de P. jirovecii con la EPOC no han obtenido resultados concluyentes sobre el tema, en particular sobre el papel que juega el microorganismo como patógeno o colonizador $[16,26]$. Datos más recientes concluyen que existe una fuerte asociación entre la colonización por $P$. jirovecii, la severidad de la obstrucción del flujo de aire y la respuesta inflamatoria en pacientes con EPOC, sugiriendo un posible papel patógeno del microorganismo en la progresión de esta enfermedad [5,20,21]. Otros estudios reportan que se puede detectar la presencia de neumocistosis en pacientes con exacerbación de la EPOC y con otras condiciones pulmonares, mediante la utilización de la inmunofluorescencia directa $[1,4,17]$. Por otra parte, la exacerbación de la EPOC también puede aumentar debido a la asociación con otras enfermedades, como hipertensión arterial, enfermedad cardiovascular, hepatopatía crónica, alcoholismo, diabetes mellitus, insuficiencia renal y deficiencias nutricionales $[22,30]$. Las enfermedades autoinmunes y del tejido conectivo, como la artritis reumatoide, son consideradas factores de riesgo para la aparición de neumocistosis. La frecuencia de la enfermedad es muy baja según algunas investigaciones $[13,35]$, pero existen estudios que han reportado la presencia de neumocistosis en pacientes con artritis reumatoide bajo tratamiento con metotrexato [29]. Todos estos datos indican que la neumocistosis debe ser sospechada en pacientes sin sida y con factores de riesgo como la EPOC [7]. En los cinco pacientes con EPOC de nuestro estudio se detectó la presencia de $P$. jirovecii, lo cual habla a favor de un excelente diagnóstico presuntivo de la enfermedad, así como de la sensibilidad de la inmunofluorescencia directa como técnica de diagnóstico. En su mayoría tenían tratamientos previos con antibacterianos de amplio espectro y anti-tuberculosos, sin haber presentado una evolución clínica favorable. A todos ellos se les suministró adicionalmente trimetoprim-sulfametoxazol, con posterior respuesta clínica satisfactoria al tratamiento. Estos resultados sugieren que $P$. jirovecii es importante en la fisiopatología de la EPOC. Sin embargo, se necesitan más estudios para definir el papel que juega este microorganismo en la progresión de esta enfermedad, así como determinar si el tratamiento para la neumocistosis puede disminuir el proceso inflamatorio en los pacientes colonizados [5].

El diagnóstico de la neumocistosis en el laboratorio depende de la identificación morfológica de $P$. jirovecii, que puede detectarse en una gran variedad de muestras respiratorias, de las cuales, el lavado bronquial, el esputo inducido y el esputo espontáneo son las más empleadas. Paradójicamente, una de las mayores dificultades en el diagnóstico de esta enfermedad es precisamente la obten- ción de una muestra adecuada, que a su vez depende de la enfermedad de base del paciente. Por otra parte, la experiencia del personal médico y de laboratorio, en la evaluación del paciente y en el procesamiento de las muestras respectivamente, juega un papel muy importante en el momento de realizar el diagnóstico $[3,9,25]$.

Es importante reconocer que la presentación clínica de la neumocistosis es diferente en los pacientes con y sin sida. La progresión de la enfermedad en pacientes con sida es subaguda y se desarrolla en el curso de una a dos semanas, con un significativo número de $P$. jirovecii en los pulmones y cantidad reducida de neutrófilos. En los pacientes sin sida, la presentación de los síntomas es variable y la enfermedad se desarrolla en pocos días, con una clínica grave acompañada de hipoxemia; como reflejo de la rapidez en la progresión de la enfermedad, la cantidad de $P$. jirovecii presente en los pulmones de los pacientes sin sida es menor y está acompañada de una reacción inflamatoria mucho mayor a expensas del aumento de la cantidad de neutrófilos [10,28,32].

El lavado bronquial se utiliza con frecuencia para el diagnóstico de neumocistosis, sobre todo en pacientes incapaces de expectorar y, particularmente, en los pacientes con cáncer [2]. Para su obtención se requiere de un broncoscopio, una técnica invasora que resulta incómoda para los pacientes, sobre todo en aquellos con infección respiratoria grave y con tendencia al sangrado, por lo que puede estar contraindicada [9]. El esputo inducido se emplea para el estudio de enfermedades respiratorias como asma, EPOC, tuberculosis, fibrosis quística, cáncer pulmonar y, por supuesto, la neumonía por $P$. jirovecii. La técnica para la obtención del esputo inducido ha facilitado la recolección de una muestra de buena calidad y en condiciones no invasoras para el paciente $[9,33]$. El esputo espontáneo es la muestra que se recibe con mayor frecuencia para realizar el diagnóstico microbiológico de las infecciones respiratorias; sin embargo, su utilización no es recomendable para el diagnóstico de la neumocistosis [28]. En nuestra experiencia, si la muestra de esputo espontáneo es procesada adecuadamente, puede llegar a tener el mismo rendimiento diagnóstico que las muestras de esputo inducido para el diagnóstico de neumocistosis en pacientes con sida $[3,7,18,25]$.

Las técnicas de laboratorio más empleadas para el diagnóstico de neumocistosis son las coloraciones de Giemsa, Gomori-Grocott, Diff-Quick, azul de toluidina y las técnicas basadas en la utilización de anticuerpos monoclonales, como la inmunofluorescencia directa. Si bien todas estás técnicas tienen valores de sensibilidad y especificidad comparables entre sí para la detección de $P$. jirovecii, siempre y cuando sean realizadas por personal con experiencia en el diagnóstico, la inmunofluorescencia directa se ha convertido en la técnica de uso preferencial $[3,9,15,18,25,28,33]$. Según los resultados de nuestro tra- 
bajo, la muestra de esputo espontáneo, procesada con la modificación estandarizada en el departamento de micología del INHRR, resultó ser una muestra útil para la detección de $P$. jirovecii en pacientes con sida, ya que al reducir su viscosidad y potenciar una mayor concentración de los microorganismos con la centrifugación, se logró obtener una muestra semejante en cualidades al esputo inducido. Con el lavado bronquial se detectó la mayor cantidad de casos de neumocistosis en pacientes con cáncer, y con el esputo inducido se detectaron casos de neumocistosis en pacientes con IRB y en pacientes con sida. En nuestro estudio, el esputo inducido resultó ser una muestra muy útil para detectar $P$. jirovecii en pacientes con IRB sin sida ni cáncer utilizando la inmunofluorescencia directa, no así el esputo espontáneo, aún con las modificaciones introducidas en su procesamiento.

Se ha reportado que la sensibilidad y la especificidad de muestras como el esputo inducido y el esputo espontáneo aumentan considerablemente con el uso de la inmunofluorescencia directa para el diagnóstico de $P$. jirovecii, al compararlas con tinciones como el Giemsa y el Grocott, inclusive cuando se usan estas muestras en poblaciones de baja prevalencia para neumocistosis, y estos resultados dependen no sólo de una toma de muestra adecuada, sino de la experiencia del laboratorio en el diagnóstico de la enfermedad $[3,9,15,18,25,28,33]$. Por otra parte, la inmunofluorescencia directa ha sido utilizada con éxito en la detección de $P$. jirovecii en personas inmunocompetentes con infecciones pulmonares crónicas [17].
Finalmente, este estudio demostró que existen diferencias en la frecuencia de la neumocistosis relacionadas con la enfermedad de base de los pacientes, y que esta enfermedad es un problema de salud importante en los pacientes inmunosuprimidos. La naturaleza retrospectiva de este trabajo y el hecho de reflejar la experiencia en el diagnóstico de un solo centro son limitaciones importantes, pero los datos obtenidos sugieren que la neumocistosis debe ser sospechada en pacientes no inmunosuprimidos con signos y síntomas de IRB, ya que suele estar acompañada de otras condiciones clínicas y que se debe ampliar el estudio de esta enfermedad en pacientes con cáncer y EPOC. La inmunofluorescencia directa es una técnica muy útil para el diagnóstico de la neumocistosis y sus resultados dependen de la evaluación clínica del paciente, una muestra tomada en óptimas condiciones y de la experiencia del personal a cargo del diagnóstico. 
1. Armbruster C, Hassl A, Kriwanek S. Pneumocystis carinii colonization in the absence of immunosuppression. Scand $\mathrm{J}$ Infect Dis 1997; 29: 591-593.

2. Báez-Escudero JL, Greene JN Sandin RL, Vincent AL. Pneumocystis carinii pneumonia in cancer patients. Abstr Hematol Oncol 2004; 7: 24-30.

3. Borelli K, Brito A, Rivas G, Panizo MM, Roldán Y. Diagnóstico de Pneumocystis carinii: Estudio comparativo entre inmunofluorescencia directa y la coloración histológica de GomoriGrocott. Bol Soc Ven Microbiol 2000; 20 : 46-52.

4. Calderón EJ, Regordan C, Medrano FJ, Ollero M, Varela JM. Pneumocystis carin infection in patients with chronic bronchial disease. Lancet 1996; 347: 977.

5. Calderón EJ, Rivero L, Respaldiza N, Morilla R, Montes-Cano MA, Friaza V, Muñoz-Lobato F, Varela JM, Medrano FJ, de la Horra C. Systemic inflammation in patients with chronic obstructive pulmonary disease who are colonized with Pneumocystis jirovecii. Clin Infect Dis 2007; 45: e17-e19.

6. Calderón EJ, Varela JM, Medrano FJ, Nieto V, González-Becerra C, Respaldiza $\mathrm{N}$, De la Horra C, Montes-Cano MA Vigil E, González de la Puente MA Cuello JA. Epidemiology of Pneumocystis carinii pneumonia in Southern Spain. Clin Microbiol Infec 2004; 10: 673-676.

7. Casanova K, Sáez A, Navas T, Reviakina V, Panizo M, Chiriboga D. Epidemiología de la neumocistosis. Medicina Interna (Caracas) 2006; 22 . Medicina

8. Cermeño JR, Hernández de Cuesta I, Alcalá F, Appice M. Pneumocystis jirovecii en centros hospitalarios del Estado Bolívar, Venezuela. Rev Biomed 2006; 17: 169-174.

9. Cruciani M, Marcati P, Malena M, Bosco O, Serpelloni G, Mengoli C Meta-analysis of diagnostic procedures for Pneumocystis carinii pneumonia in HIV-infected patients. Eur Respir J 2002 20: 982-989.

10. Festic E, Gajic O, Limper $A H$, Aksamit TR. Acute respiratory failure due to Pneumocystis pneumonia in patients without human immunodeficiency virus infection. Outcome and associated
features. Chest 2005; 128: 573-579.

11. Fisk D, Meshnick S, Kazanjian P. Pneumocystis carinii pneumonia in patients in the developing world who patients in the developing world who syndrome. Clin Infect Dis 2003; 36 : syndrom
$70-78$.

12. Gill VJ, Evans G, Stock F, Parrillo JE, Masur H, Kovacs JA. Detection of Pneumocystis carinii by fluorescent antibody satin using a combination of three monoclonal antibodies. J Clin Microbiol 1987; 25: 1837-1840.

13. Godeau B, Coutant-Perronne V, Huoung DLT, Guillevin L, Magadur G De Bandt M, Dellion S, Rossert J, Rostoker G, Piette JC, Schaeffer A. Pneumocystis carinii pneumonia in the course of connective tissue disease: report of 34 cases. J Rheumatol 1994: 21: 246-251.
14. Hui M, Kwok WT. Pneumocystis carinii pneumonia in Hong Kong: a 10 year retrospective study. J Med Microbio 2006; 55: 85-88.

15. Kovacs JA, Ng VL, Masur H, Leoung G Hadley WK, Evans G, Lane HC Ognibene FP, Shelhamer J, Parrillo JE, Gill VJ. Diagnosis of Pneumocystis carinii pneumonia: improved detection in pneumonia: improved detection sputum with use of monoclonal 589-593.

16. Maskell NA, Waine DJ, Lindley A, Pepperell JC, Wakefield AE, Miller RF, Davies RJ. Asymptomatic carriage of Pneumocystis jirovecii in subjects undergoing bronchoscopy: a prospective undergoing bronchoscopy: a prosp

17. Medrano FJ, Montes-Cano M, Conde M, de la Horra C, Respaldiza N, Gasch A, Perez-Lozano MJ, Varela JM, Calderón EJ. Pneumocystis jirovecii in general population. Emerg Infect Dis 2005; 11: 245-250.

18. Metersky ML, Aslenzadeh J, Stelmach P. A comparison of induced and expectorated sputum for the diagnosis of Pneumocystis carinii pneumonia. Chest 1998; 113: 1555-1559.

19. Morris A, Lundgren JD, Masur $\mathrm{H}$, Walzer PD, Hanson DL, Frederick T, Huang L, Beard CB, Kaplan JE. Current epidemiology of Pneumocystis pneumonia. Emerg Infect Dis 2004; 10: 1713-1720.

20. Morris A, Sciurba FC, Lebedeva IP Githaiga A, Elliot WM, Hogg JC Huang L, Norris KA. Association of chronic obstructive pulmonary disease chronic obstructive pulmonary disease severity and Pneumocystis colonization. 408-413.

21. Morris A, Sciurba FC, Norris KA. Pneumocystis: a novel pathogen in chronic obstructive pulmonary disease? COPD 2008; 5: 43-51.

22. Naresh $A$, Dewan MB, Rafique $S$ Badar K. Acute exacerbation of COPD: factors associated with poor treatment outcome. Chest 2000; 177: 662-671.

23. Nittayananta $\mathrm{W}$, Chanowanna N, Winn T, Silpapojakul K, Rodklai A

Jaruratanasirikul S, Liewchampatana K. Co-existence between oral lesions and opportunistic systemic diseases among HIV-infected subjects in Thailand. J Ora Pathol Med 2002; 31: 163-168.

24. Pagano L, Fianchi L, Mele L, Girmenia C, Offidani M, Ricci P, Mitra ME, Picardi M, Caramatti C, Piccaluga P, Nosari A, Buelli M, Allione B, Cortelezzi A, Fabbiano F, Milone G, Invernizzi R, Martino B, Masini L, Todeschini G, Martino B, Masini L, Todeschini G, Martino P, Del Favero A. Pneumocystis carinii pneumonia in patients with malignant haematological diseases: 10 years' experience of infection in GIMEMA centers. Brit J Haematol 2002 117: 379-386.
25. Panizo M Reviákina V Vázquez C Diagnóstico de Pneumocystis carinii por inmunofluorescencia directa modificada y coloración de Gomori-Grocott. Estudio comparativo. Bol Soc Ven Microbio 2000; 20: 98-103.

26. Probst $M$, Ries $H$, Schmidt-Wieland $T$, Serr A. Detection of Pneumocystis carini DNA in patients with chronic lung diseases. Eur J Clin Microbiol Infect Dis 2000; 19: 644-645.

27. Roblot F, Le Moal G, Godet C, Hutin P, Texereau M, Boyer E, Prazuck T, Lacroix C, Souala MF, Raffi F, Weinbreck P, Besnier JM, Garo B, de Gentile L, Becq-Giraudon B. Pneumocystis carinii pneumonia in patients with hematologic malignancies: a descriptive study. J Infect 2003; 47: 19-27.

28. Rodriguez M, Fishman JA. Prevention of infection due to Pneumocystis spp. in human immunodeficiency virus-negative immunocompromised patients. Clin Microbiol Rev 2004; 17: 770-782.

29. Roux N, Flipo RM, Cortet B, Lafitte JJ, Tonnel AB, Duquesnoy B, Delcambre B. Pneumocystis carinii pneumonia in rheumatoid arthritis patient treated with methotrexate. A report of two cases. Rev Rhum Engl Ed 1996; 63: 453-456.

30. Sat S, Windom E, Marrades RM. Chronic obstructive pulmonary disease. Thorax 2001; 55: 735-748.

31. Sepkowitz KA, Brown AE, Telzak EE, Gottlieb S, Armstrong D. Pneumocystis carinii pneumonia among patients withou 267: 832-837.

32. Thomas CF Jr, Limper AH. Pneumocystis pneumonia. N Engl J Med 2004; 350: 2487-2498.

33. Turner D, Schwarz Y, Yust I. Induced sputum for diagnosing Pneumocystis carinii pneumonia in HIV patients: new data, new issues. Eur Respir J 2003; 21 : 204-208.

34. Uno K, Konishi M, Yoshimoto $\mathrm{E}$ Kasahara K, Mori K, Maeda K, Ishida E, Konishi N, Murakawa K, Mikasa K. Fatal cytomegalovirus-associated adrenal insufficiency in an AIDS patient receiving corticosteroid therapy. Intern Med 2007: 46: 617-620.

35. Ward MM, Donald F. Pneumocystis carinii pneumonia in patients with connective tissue diseases. Arthritis Rheum 1999; 42: 780-789. 